\title{
An Analysis of Game-Related Emotions Using EMOTIV EPOC
}

\author{
Jerzy Kosiński, Krzysztof Szklanny \\ Polish-Japanese Academy of Information \\ Technology ul. Koszykowa 86, 02-008 \\ Warsaw, Poland \\ Email: \{s9347, kszklanny\}@pjwstk.edu.pl\}
}

\author{
Alicja Wieczorkowska \\ Polish-Japanese Academy of \\ Information ul. Koszykowa 86, \\ 02-008 Warsaw, Poland \\ Email: alicja@poljap.edu.pl
}

\author{
Marcin Wichrowski \\ Polish-Japanese Academy of \\ Information Technology ul. \\ Koszykowa 86, 02-008 Warsaw, \\ Poland. Email: \\ mati@pjwstk.edu.pl
}

\begin{abstract}
Computer games represent a very popular form of entertainment. Therefore, playing games became an object of interest for researchers. The research on the brain activity of players when playing a game is an experimental contribution to the neurophysiology of the central nervous system, and it also supports marketing research.

Devices that register electromagnetic waves generated by the brain, e.g. EEG (Electroencephalography) can be used by psychologists studying the impact of games on users when the game. Our goal was to analyze emotion changes while playing video games, based on EEG signal registered with EMOTIV EPOC headset, and identify the strongest emotions accompanying the game. We also wanted to link emotions to particular elements of the game. Game developers, especially educational and therapeutic, can use the outcomes of this work in the practical implementation of the brain-computer interfaces in their products, in order to create better and more engaging games.
\end{abstract}

\section{INTRODUCTION}

$\mathrm{E}$ LECTROENCELOGRAPHY (EEG) is a noninvasive examination of brain function, using electrodes attached to the scalp. These electrodes record the electric field (electric potential) generated by the brain. Analysis of EEG records provides information on the activity of specific areas of the brain. EEG examinations are performed routinely as part of neurological diagnostics of the central nervous system. An important medical application of EEG is the diagnosis of coma and of brain death. EEG is also used as a tool in neurophysiological and psychoneurological tests, for example during the examination of the level of anesthesia, in the study of sleep disorders [1], and the analysis of the effects of drugs on the central nervous system. EEG is also used in neurophysiological research, including studies of cognitive and emotional processes. The emergence of instruments enabling low-cost registration of EEG signals contributes to the increase of interest in the use of such measurements in various fields of study.

The possibility of registering brain activity is also attractive in areas far from medicine. Unlike routine medical

This work was partially supported by the Research Center of PJAIT, supported by the Ministry of Science and Higher Education in Poland procedures, the research on brain activity does not focus on detecting anomalies that may indicate pathological changes, but on linking the recorded activity of the brain with received stimuli, cognitive activities, progress in training various skills [2], degree of relaxation, etc.

As a non-invasive method, and also more widely available recently, EEG is a natural candidate for use in the work on the brain-computer interfaces (BCI). In this work we will discuss some of the results of research in this area.

Game developers, especially educational and therapeutic, are particularly interested in the practical implementation of $\mathrm{BCI}$ in their products. EEG registering the reaction of players and the emotions accompanying the game is an interesting source of information for game designers, striving to create a game model that is satisfying for each user. EEG tests are also used by psychologists in studies on the impact of games on users.

\section{CONSUMER EEG DEVICES}

Standard EEG measurements are performed using a stationary device, in an outpatient or laboratory setting. At present, various solutions are available on the market that allow recording EEG signals using portable devices [3], [4], [5]. Since these devices are inexpensive and easy to use, they can be applied in many areas, including using them as BCI interfaces in consumer applications. The usability of commercial EEG devices was tested in [6].

The EMOTIV EPOC + wireless headset provides 14 channel EEG, plus 2 reference channels. Saline based wet sensors allow avoiding sticky gels. The headset is quite flexible, but its plastic structure limits the ability to adapt the device to untypical sizes or the shapes of the head. The signal is sent through Bluetooth or proprietary wireless communication. Battery life is up to 12 hours when using proprietary wireless, and up to 6 hours when using Bluetooth.

\section{EMOTIV EPOC IN APPLICATIONS AND RESEARCH}

The EMOTIV hardware and software evoked great interest. The authors in [7] compared EPOC with the research EEG system (Neuroscan Synamps) for measuring 
auditory event-related potentials. Their findings suggest that EPOC compare well with Synamps in such tests, and EPOC is easier to use because of its quick and clean set-up. On the other hand, EPOC may perform significantly worse than a medical device, as shown in [8]. The authors of [8] tested the ANT medical grade system and EPOC on P300 responses. P300 is an involuntary positive potential that is evoked about $300 \mathrm{~ms}$ after the user has perceived a relevant and rare stimulus. EPOC indicated lower SNR (signal-to-noise ratio), so the authors suggested choosing EPOC only for noncritical applications such as games, as it is not reliable enough for medical purposes such as prosthesis control.

\section{EXPERIMENT DESIGN}

The aim of the experiment was to register EEG of players while playing computer games, and analyze emotions, depending on the type of game. During testing, EEG of 15 males aged 23-28 were registered. Persons who often play computer games were selected for our experiments.

Two computers were used in the experiments. The EMOTIV headset was connected to one of them and EEG data were recorded. Only the experimenter had access to this computer. The second computer was used for gaming, and this computer was used by the players. Three games with different gameplay characteristics have been selected for these experiments.

At the beginning of each experiment session, the experimenter puts the EPOC headset on the player's head. Next, the experiment adjusts the headset while checking the readings from EPOC, to assure the best possible quality of the signal. Afterwards, the experiment session is performed. The session consists of 7 parts. The player is informed before each part what he is expected to do, and how long it will take. These 7 parts (stages) of the experiment session are listed below:

1. Initial EEG measurements - the tested player closes his eyes, tries to relax, and not think about anything. The purpose of this stage of the experiment is to register EEG readings from the resting-state of the brain, for comparison with reading acquired in the next stages of the experiment.

2. Square game - the player plays the Square game, which is a team based game. His activity is monotonous, neither requiring mental effort nor psychically engaging. The registered EEG are to reflect moderate brain activity, typical of normal computer use.

3. Rocket League played with bots - the test player plays a 5 minute match with a computer-controlled player (bot).

4. Rocket League played online - the player plays a 5 minute match with other players (persons) over the Internet.

5. Watching high level game - the player watches a 5 minute match of the Rocket League, played by advanced players.

6. Super Bomberman game against bots - the player plays 3 matches of the Super Bomberman game against bots.
7. Super Bomberman game with another player - the test player plays a match of the Super Bomberman game with another player (person) against 2 bots.

As mentioned before, we selected 3 games of different characteristics. EEG readings were acquired using two methods. In the first method, each 0.5 the average power of theta, alpha, beta, and gamma waves was acquired (for beta waves, separately for low and high subbands). The wave ranges are as follows: theta $4-8 \mathrm{~Hz}$, alpha $8-13 \mathrm{~Hz}$, beta 13 30 (low 13-20 Hz, high 21-30 Hz), gamma 30-80 Hz.

The second method consisted in collecting emotion data using Emotiv software, for the following emotions: boredom, meditation, frustration, instantaneous excitement, and longterm excitement, scaled from 0 to 1 . The Emotiv software is needed to acquire emotion data, facial expressions, and access mental commands.

After the preliminary analysis of the collected emotion data in the first round of tests, we decided to reject boredom and meditation, because of very low level of the obtained signals.

We also experienced technical problems with the EPOC headset we used, as 2 electrodes, A3 and AF3, did not work properly (and the readings from these electrodes had to be discarded, as they represented noise only). Therefore, we had to replace this headset with another one. This shows that this equipment is delicate and prone to failures. Accessories for EPOC are available, but it takes time to obtain these accessories.

\section{RESUlTS}

In our research, we describe emotions in 2-dimensional valence/arousal plane [9]. Based on the readings from the EPOC electrodes, we can calculated valence values, indicating positive or negative emotional states, and estimate arousal levels. Since beta to alpha ratio is a reasonable indicator of arousal state [10], we estimate arousal index $E_{A}$, as the indicator of arousal state, according to the following formula:

$$
E_{A}=\frac{E_{\beta}}{E_{\alpha}},
$$

$E_{\beta}$ - average beta power, $E_{\alpha}$ - average alpha power.

The valence index can be calculated for a particular electrode, or brain regions. Typically, this index is calculated for frontal $(\mathrm{F})$ and antero-frontal (AF) electrodes, as beta and alpha waves are most pronounced and can be most easily measured in the frontal and the middle part of the brain [11]. Therefore, data from A and AF electrodes are the best choice when estimating valence [12].

It is suggested that greater left frontal activity is associated with positive affect and/or approach motivation, and that greater right frontal activity is associated with negative affect and/or withdrawal motivation, although in [13] the author found that the frontal asymmetry is responsive to 
motivational direction and not affective valence. Still, since we had symmetrically placed electrodes at our disposal, we decided to use the following measure of valence $E_{V}$, calculated by comparing the alpha power and beta power between the right and left hemispheres [10]:

$$
E_{V}=\frac{E_{\alpha}^{r}}{E_{\beta}^{r}}-\frac{E_{\alpha}^{l}}{E_{\beta}^{l}},
$$

$\mathrm{r}$ - right hemisphere,

1 - left hemisphere.

$E_{V}$ was calculated for the readings of symmetrically placed electrodes, or for averaged power calculated over selected brain parts. Positive $E_{V}$ values indicate positive emotions, and negative values indicate negative emotions.

In the presented graphs we illustrate outcomes for the data collected in the $3^{\text {rd }}$ stage of experiment, i.e. when playing Rocket League with bots:

- $E_{V}$ for readings from electrodes $\mathrm{F} 3, \mathrm{AF} 3$ and also $\mathrm{T} 7$ for $E_{\alpha}^{l}$ and $E_{\beta}^{l}$, and readings from electrodes $\mathrm{F} 4, \mathrm{AF} 4$ and also T8 for $E_{\alpha}^{r}$ and $E_{\beta}^{r}$

- Instantaneous Excitement calculated using Emotiv software

- arousal index $E_{A}$ for readings calculated for frontal parts of the brain

\begin{tabular}{|c|l|l|l|l|l|l|l|}
\hline & Stimulus & $\begin{array}{l}\text { Avg. } \\
\text { Ex. }\end{array}$ & $\begin{array}{l}\text { Ex. } \\
\text { var. }\end{array}$ & Avg.Fr. & $\begin{array}{l}\text { Fr. } \\
\text { var. }\end{array}$ & TE & TF \\
\hline 1 & Meditation & 0.541 & 0.153 & 0.541 & 0.09 & $12 \%$ & $3 \%$ \\
\hline 2 & Squares & 0.549 & 0.207 & 0.5 & 0.127 & $16 \%$ & $4 \%$ \\
\hline 3 & $\begin{array}{l}\text { "Rocket } \\
\text { League", bots }\end{array}$ & 0.564 & 0.244 & 0.526 & 0.186 & $21 \%$ & $10 \%$ \\
\hline 4 & $\begin{array}{l}\text { "Rocket } \\
\text { League", } \\
\text { persons }\end{array}$ & 0.504 & 0.228 & 0.512 & 0.168 & $14 \%$ & $8 \%$ \\
\hline 5 & $\begin{array}{l}\text { Watching } \\
\text { "Rocket } \\
\text { League" }\end{array}$ & 0.518 & 0.266 & 0.483 & 0.153 & $20 \%$ & $3 \%$ \\
\hline 6 & $\begin{array}{l}\text { "Super } \\
\text { Bomberman”, } \\
\text { bots }\end{array}$ & 0.621 & 0.2 & 0.568 & 0.144 & $22 \%$ & $7 \%$ \\
\hline 7 & $\begin{array}{l}\text { "Super } \\
\text { Bomberman”, } \\
\text { persons }\end{array}$ & 0.579 & 0.224 & 0.537 & 0.153 & $23 \%$ & $7 \%$ \\
\hline
\end{tabular}

Avg. Ex - Average Excitation; Ex. var. - Excitation variance; Avg. Fr. Average Frustration Fr. var.- Frustration variance; TE - \% time with excitation $>0.8 ; \mathrm{TF}-\%$ time with frustration $>0.8$

We chose F3 and F4 as suggested in [10], and we additionally chose another symmetrically placed pair, AF3 and AF4, located close to F3 and F4. Additionally, electrodes T7 and T8 (temporal part of the brain) were chosen, as suggested in [14], where the authors indicate that information from the temporal part of the brain is significant in emotion classification.

The comparison of $E_{V}$ values for various pairs of electrodes, i.e. F3 and F4, AF3 and AF4, T7 and T8, shows differences in brain activity for frontal and temporal parts of the brain.

Instantaneous Excitement calculated using Emotiv software shows large variability, and the results are directly related to game events.

The valence measure $E_{V}$ and the arousal index $E_{A}$ for frontal electrodes are not related directly to particular game events. The calculated values do not indicate stimulate from the game.

Therefore, we decided to use Emotiv software in further investigations. Instantaneous Excitement for the game and Long-Term Excitement for meditation show, that Excitement is related to game events.

The assessment of the emotion readings was done on the basis of average Instantaneous Excitement and Frustration, as well as Meditation and Boredom, calculated using Emotiv software. Average values and variance, as well as the percentage of time when Frustration or Excitation level was high (above 0.8) are shown in Tab. I. Initially, we also calculated these values for the Emotiv headset with damaged electrodes, but the results were inconsistent, so we decided to use another headset.

The values were averaged over all test participants, separately for each stage of the experiment.

As we can see, our experiment consisted of non-engaging (mentally, technically) as well as of highly engaging parts. Meditation, playing Square, or watching a game was rather non-engaging, whereas active playing games requiring high mental and motoric involvement (Rocket League, Super Bomberman) was highly demanding.

Excitement results show that average Excitation and the time of high Excitement when playing with other persons are greater than when playing with bots. For less engaging activities, the test results are similar, but variance is lower.

Frustration remained approximately constant during engaging activities, but variance increases when playing Rocket League with other persons, compared to playing with bots. Frustration results for non-engaging activities are a bit surprising; this is in line with results obtained in other investigations.

The highest meditation level was obtained for mentally non-engaging activities, but the difference in meditation level is small.

The highest boredom level was obtained when watching Rocket League match. This agrees with the relations of the tested players, as they emphasized their boredom during this part of the experiment.

Emotion levels when playing Rocket League match and EV values calculated for various electrodes are shown in Fig. 1. Unfortunately, the results from Emotive software are not perfect neither, as some exciting parts of the match are not reflected with the Excitement graph. For instance, only slight emotion changes can be observed between $180^{\text {th }}$ and $230^{\text {th }}$ second of the match, and rapid change can be seen during the scored goal only, whereas during this part of the match the ball was very close to the goal, and the player shot several times. After the $320^{\text {th }}$ second of the match the excitement 
suddenly drops almost to zero, whereas it is not reflected in the game, as the player was constantly defending goal during this part of the match. Frustration graph shows similar changes, but frustrating actions like loosing goal do not evoke frustration in this graph. In the final part of the match both scoring and defending goal correspond to surprisingly high frustration in this graph. The meditation and boredom levels also do not correspond to the gameplay.

The comparison of meditation and boredom levels registered in various experiments shows that these values are averaged over long runs. Therefore, such results are not very useful for estimating emotions while playing computer games.

\section{DISCUSSION}

Our experiments confirm that playing a game causes brain activity changes, and these changes can be associated with strong emotions evoked by some parts of the gameplay. Similar observations were made in [16], with the focus on the changes of brainwaves power during high intensity and low intensity game events.

The analysis of the acquired data shows that the equipment and software are not sufficient to identify gameevoked emotions.

The literature we analyzed suggests that satisfactory results might be achieved only after complicated analysis and processing of the EEG data [17][18][19]. The use of neural networks is suggested. Deep neural networks are very often used recently, but since neural nets require large amount of data, are data are not sufficient for such analysis.

\section{SUMMARY AND CONCLUSIONS}

The Emotiv software quite successfully detects excitement and frustration. Still, it is difficult to assure if the readings were accurate enough. The analysis of video recordings of the experiments is necessary to assess which parts of the gameplay correspond to the observed changes of emotions, and how to interpret these changes.

The algorithms implemented in the Emotiv software are not publicly available. Therefore, we cannot verify directly how various emotions are processed by these algorithms and what readings are reported for particular emotions. The values returned by these algorithms as meditation level (Meditation) and boredom level (Boredom) do not show large variability. The returned levels of excitement and frustration present larger variability, related to the gameplay as expected, but for each of these readings we can find parts of the play when these readings do not reflected emotions associated with playing the game. Since we cannot access these algorithms, nor formulas for calculating the readings, we are not able to assess whether the obtained values are correct or random. Similar problem was described by Harrison in [20].

The analysis of literature on emotion identification shows problems which must be addressed in order to achieve efficient detection and identification of emotions while playing computer games, based on EEG data. One of the issues to solve is to objectively identify game-related emotions. Various persons subjected to similar stimuli may experience different emotions. In the case of computer games, the player preferences may influence the experienced emotions. In the literature in the psychology domain [9] difficulties in the objective classification of emotions were reported, as the subjects may attribute various meaning to the same labels of emotional states, so the reported emotions may be ambiguous.

The method of evoking emotions and the emotion time should also be taken into account, no matter what emotion

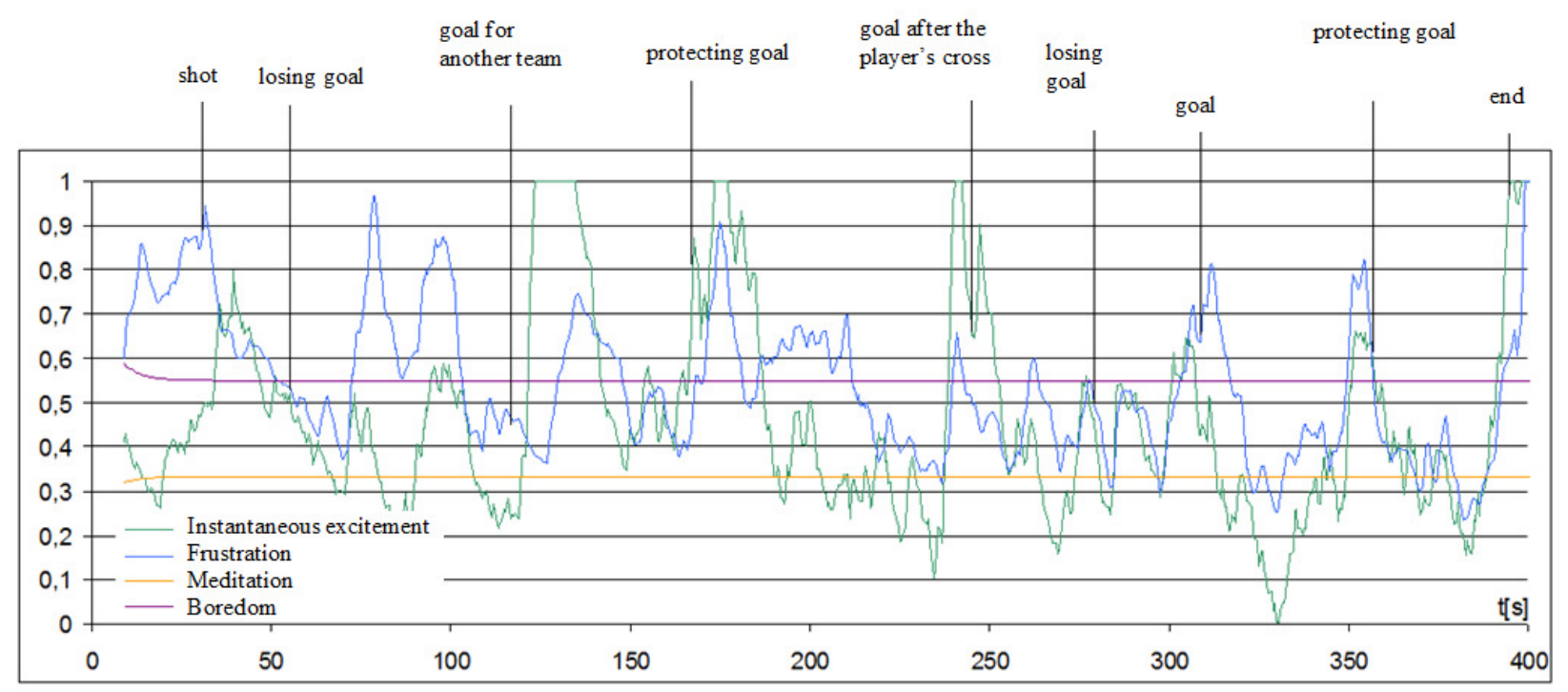

Fig.1. Emotions during the Rocket League match, calculated using Emotiv software 
classification method was applied.

Depending on the type of stimulus and other experiment conditions, the registered EEG may represent emotional states of various intensity, duration, and co-occurrence of motor or cognitive activity. Even successful classification of emotional states, based on EEG data, can be less accurate for other stimuli or experiment conditions.

Computer games represent quite a specific type of stimuli, evoking emotions. Various types of gameplay may evoke different emotions in various players; the intensity of these emotions may also vary between players. In the case of typical computer games, intensive cognitive activity accompanies the playing, and at least medium motor activity. The player has to understand the gameplay, and actively control the game by means of the appropriate controller (at least until BCI interfaces are not efficiently and broadly implemented as game controllers). In typical games, there are numerous unforeseen events, to avoid experiencing boredom. Therefore, intensive emotions are expected, and they must quickly change in time.

As mentioned above, we cannot rely on the emotions reported by players when we want to analyze their emotions experienced while playing a computer game. This was reported by Salminen and Ravaja in [15], as well as McMahan et al. in [16]. Also, negative emotions experienced while playing, e.g. fear or frustration, cause experiencing positive emotion of satisfaction at the end of the game, which is specificity of computer games. The player is aware of the game convention and he or she is distanced to the experienced emotion, so the player subjectively assesses emotions as less intense. Without further investigations it is difficult to answer the question whether these differences can be seen in EEG.

Depending on the type and dynamics of the game, and also on the type of emotions to be analyzed, the analysis should be performed on shorter or longer time segments. The analysis of the power of brainwaves assumes averaging of the registered potentials, both over time and over frequency. In our experiment, we registered 2 readings of the Emotiv EPOC headset per second. Therefore, we can perform the analysis of psycho-neurological phenomena of medium duration. In the literature, short time EEG readings are also reported, i.e. of order of hundreds of readings per second, also in investigations on emotion detection. The Emotiv EPOC headset can also provide such data, when using appropriate software library [7], [8]. However, such analysis requires much more complicated experiment planning, as precise synchronization of the EEG readings with stimuli is necessary [19]. Additionally, in order to obtain high signal to noise ratio, numerous repetitions of stimuli are necessary. On the other hand, quick repetition of similar stimuli should be avoided. Therefore, such experiments are behind the topic of this work for now.

\section{REFERENCES}

[1] E. Weaver, M. Gradisar, H. Dohnt, N. Lovato, and P. Douglas, "The effect of presleep video-game playing on adolescent sleep," Journal of Clinical Sleep Medicine, vol. 6, 2010, pp. 184-189.

[2] S. Wolf, E. Brölz, D. Scholz, A. Ramos-Murguialday, P. Keune, M. Hautzinger, N. Birbaumer, and U. Strehl, „Winning the game: brain processes in expert, young elite and amateur table tennis players", Frontiers in Behavioral Neuroscience, vol. 8, 2014, pp. 11-12.

[3] T. B. Cedro, and A. Grzanka, "CeDeROM Brain Computer Interface", Information Technologies in Biomedicine. Springer LNCS 7339, 2012, pp. 219-231.

[4] W. D. Hairston, K. W. Whitaker, A. J. Ries, J. M. Vettel, J. C. Bradford, and S. E. Kerick, K. McDowell, "Usability of four commercially-oriented EEG systems," Journal of Neural Engineering, vol. 11, 2014, pp. 1-14.

[5] T. S. Grummett, R. E. Leibbrandt, T. W. Lewis, D. DeLosAngeles, D. M. W. Powers, J. O. Willoughby, K. J. Pope, and S. P. Fitzgibbon, "Measurement of neural signals from inexpensive, wireless and dry EEG systems," Physiological Measurement, vol. 36, 2015, pp. 14691484.

[6] W. D. Hairston, K. W. Whitaker, A. J Ries, J. M. Vettel, J. C. Bradford, S. E. Kerick, and K. McDowell, J. Neural Eng., vol. 11 046018, 2014.

[7] N. A. Badcock, P. Mousikou, Y. Mahajan, P. de Lissa, J. Thie, and G. McArthur, "Validation of the Emotiv EPOC EEG gaming system for measuring research quality auditory ERPs", PeerJ 1:e38, 2013

[8] M. Duvinage, T. Castermans, M. Petieau, T. Hoellinger, G. Cheron, and T. Dutoit, "Performance of the Emotiv Epoc headset for P300based applications", BioMedical Engineering OnLine, vol. 12, no. 56, 2013.

[9] J. A. Russell, “A circumplex model of affect," J. Pers. Soc. Psychol., vol. 39, no. 6, pp. 1161-1178, 1980.

[10] R. Ramirez and Z. Vamvakousis, "Detecting emotion from EEG signals using the Emotive Epoc device," in Brain Informatics, LNCS vol. 7670 LNAI, 2012, pp. 175-184.

[11] T. Matlovič, "Emotion Detection using EPOC EEG device", in IIT SRC 2016, available at https://www.pewe.sk/wpcontent/uploads/2016/01/20_iitsrc_matlovic.pdf

[12] Y. Liu and O. Sourina, "Real-time subject-dependent EEG-based emotion recognition algorithm," in Lecture Notes in Computer Science (including subseries Lecture Notes in Artificial Intelligence and Lecture Notes in Bioinformatics), 2014, vol. 8490, pp. 199-223.

[13] E. Harmon-Jones, "Clarifying the emotive functions of asymmetrical frontal cortical activity”, Psychophysiology, vol. 40, pp. 838-848, 2003.

[14] W. L. Zheng and B. L. Lu, "Investigating Critical Frequency Bands and Channels for EEG-Based Emotion Recognition with Deep Neural Networks," IEEE Trans. Auton. Ment. Dev., vol. 7, no. 3, pp. 162175,2015

[15] M. Salminen and N. Ravaja, "Increased oscillatory theta activation evoked by violent digital game events," Neurosci. Lett., vol. 435, no. 1, pp. 69-72, 2008.

[16] T. McMahan, I. Parberry, and T. D. Parsons, "Modality specific assessment of video game player's experience using the Emotiv,' Entertainment Computing, vol. 7, pp. 1-6, 2015.

[17] N. A. Badcock, P. Mousikou, Y. Mahajan, P. de Lissa, J. Thie, and G. McArthur, "Validation of the Emotiv EPOC ${ }^{\circledR}$ EEG gaming system for measuring research quality auditory ERPs," PeerJ, vol. 1, p. e38, 2013.

[18] M. Duvinage, T. Castermans, M. Petieau, T. Hoellinger, G. Cheron, and T. Dutoit, "Performance of the Emotiv Epoc headset for P300based applications," Biomed. Eng. Online, vol. 12, no. 1, 2013.

[19] A. Sivanathan, T. Lim, S. Louchart, and J. Ritchie, "Temporal multimodal data synchronisation for the analysis of a game driving task using EEG," Entertain. Comput., vol. 5, no. 4, pp. 323-334, 2014.

[20] T. Harrison, The Emotiv mind: Investigating the accuracy of the Emotiv EPOC in identifying emotions and its use in an Intelligent Tutoring System. Department of Computer Science and Software Engineering, University of Canterbury 2013 